\title{
Design of a Controlled-Release Ergometer FOR THE MEASUREMENT OF MusculotendinOUS STIFFness OF THE KNEE Flexors
}

\author{
Eric L. Dugan, ${ }^{1}$ Robert U. Newton, ${ }^{2}$ Ttm L. A. Doyle, ${ }^{2}$ and Brendan Humphries ${ }^{1}$ \\ ${ }^{1}$ Biomechanics Laboratory, Ball State University, Muncie, Indiana 47306; ${ }^{2}$ School of Biomedical and Sports \\ Sciences, Edith Cowan University, Perth, WA 6027, Australia.
}

Aastract, Dugan, E.L., R.U. Newton, T.L.A. Doyle, and B. Humphries. Design of a controlled-release ergometer for the measurement of musculotendinous stiffness of the knee flexors. J. Strength Cond. Res. 19(4):959-963. 2005.-The stiffness of muscle-tendon units (MTUs) influences many aspects of human movement from athletic performance to injury risk. Presently the controlled-release technique of measuring MTU stiffness has been applied almost exclusively to the distal joints of the body, i.e., the ankle. This is primarily because of the mechanical limitations of implementing this technique. However, in order to better understand how the elastic properties of the MTU affect both performance and injury potential, measurements of MTU stiffness of the more proximal joints must be made. The knee flexors are a logical choice because of the integral role of MTU stiffness of this muscle group in both hamstring strains and knee injury. The purpose of this study was to modify a commercial ergometer so that it could be used to measure the musculotendinous stiffness of the knee flexors. Data are presented for a representative participant to illustrate the feasibility and capability of this ergometer, and the measured MTU stiffness was $519 \mathrm{~N} \cdot \mathrm{m}^{\mathrm{rad}} \mathrm{rad}^{-1}$ at a knee flexion moment of $100 \mathrm{~N} \cdot \mathrm{m}$. Our results indicate that it is indeed possible to modify a commercial ergometer and measure musculotendinous stiffness of large muscle groups crossing proximal joints.

KEY WoRDs. stiffness, elasticity, muscle, hamstrings, ergometer

\section{INTRODUCTION}

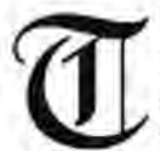

he stiffness of muscle-tendon units (MTUs) influences many aspects of human motion $(2,7$, $8,13)$. This mechanical characteristic has considerable importance for athletic performance in both maximum strength capability and power output $(13,14)$. Further, there is emerging evidence that MTU stiffness is a factor in various injury mechanisms, particularly in the common injuries of anterior cruciate ligament (ACL) rupture $(11,12)$ and hamstring damage (10). If MTU stiffness could be easily and reliably assessed, this would open up an important avenue for scientific investigation. The effects of training programs for performance enhancement and/or injury reduction could be evaluated to provide valuable knowledge for the strength and conditioning specialist.

The measurement of musculotendinous stiffness in vivo is a complex task. There is a wide range of techniques currently used to estimate the "stiffness" of various joints and MTUs that are based on different assumptions of muscle-tendon behavior and working definitions of stiffness; the reader is referred to the review by Latash and Zatsiorsky (9). One method of measuring musculotendinous stiffness, specifically stiffness of the series elas- tic component (SEC), is the controlled-release technique. The definition of stiffness used in this technique, unlike the definition used in others, is in good agreement with the definition used in physics, in which stiffness is a material property of objects capable of generating an opposing force against an externally applied force as well as storing and releasing elastic energy (9). The controlledrelease technique is designed to measure this stored elastic energy of the SEC.

In short, the controlled-release technique involves shortening the muscle group in question at a constant, high speed and then measuring the decline in torque as a function of angle. This decline in torque represents the elastic forces of the SEC and thus can be used to determine the stiffness of the SEC. Although this experimental design has been utilized successfully by several groups of investigators to quantify the elastic properties of the SEC of the ankle joint (3-6), to our knowledge this technique has not been adapted for the measurement of musculotendinous stiffness of other joints. There are several reasons for this: (a) a specialized ergometer is needed to perform such measurements; (b) it is more difficult to correct for the inertial moment produced by the acceleration of larger body segments, i.e., the lower leg and foot; and (c) the complexity of the correction procedures and data processing as proposed by de Zee and Voigt (3) and Hof (5).

The purpose of the present study was to determine the feasibility of modifying a commercially available ergometer to measure the series elasticity of the knee flexors using a controlled-release protocol as described by de Zee and Voigt (3) and Hof (4-6). The underlying assumptions were that a commercial ergometer could in fact be modified to meet the mechanical requirements of the controlled-release protocol and that SEC stiffness of the knee flexors was measurable with this technique. The significance of this study lies in the potential for this type of ergometer to be used in screening protocols to assess injury risk and design appropriate training protocols for athletes of all levels. The following is a "technical note" describing our efforts to develop such an ergometer.

\section{Methods}

In order to implement this technique to measure musculotendinous stiffness of the knee flexors, a machine capable of moving the lower leg at high, constant velocities was necessary. There are 2 distinct challenges associated with this task: (a) modifying a commercial ergometer to produce the needed movement, and (b) attaching the lower leg to the ergometer. 


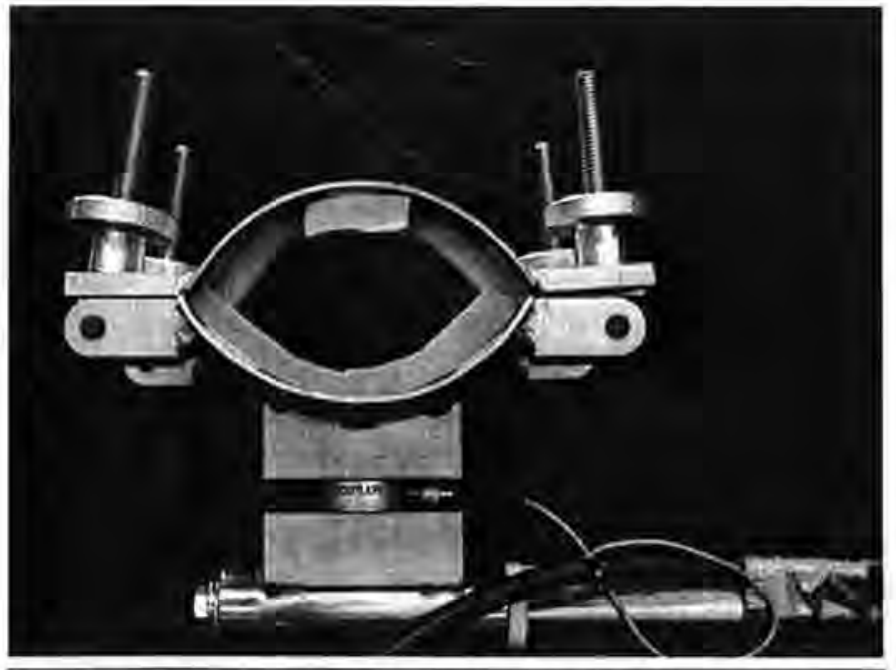

Figure 1. Instrumented leg cuff used in conjunction with ergrometer.

The ergometer available for use was a Cybex NORM (CSMI, Norwood, MA) isokinetic ergometer. We determined that the ergometer must be capable of moving the lower leg at velocities greater than $6 \mathrm{rad} \cdot \mathrm{s}^{-1}$. This value was based on measured maximum velocities of unloaded knee flexion, which were found to be between 5,1 and 5.7 $\mathrm{rad} \cdot \mathrm{s}^{-1}$. In its standard or "factory" condition, the Cybex NORM is not capable of producing velocities of this magnitude. However, this velocity constraint is a softwareimplemented limitation and not hardware-based. Therefore, in order to utilize the Cybex, a custom program was developed using Labview (National Instruments, Austin, TX) that in conjunction with a personal computer and an AT-MIO-16E-1 data acquisition card (National Instruments) could be used to control the BRU-200 drive module (Electro-Craft; Reliance Motion Control, Inc., Eden Prairie, MN) of the Cybex and consequently the motor. Through use of the custom software, the maximum speed of the ergometer was increased to approximately 7.5 $\mathrm{rad} \cdot \mathrm{s}^{-1}$, which exceeds the measured maximum velocity of unloaded knee flexion by $25-30 \%$.

In addition to modifying the ergometer's capability to produce high velocities, a suitable machine-leg interface had to be designed. In order for the controlled-release protocol to be successful, the leg must be attached to the ergometer in such a way that the leg would have the same velocity profile as the ergometer. A specialized cuff was designed that clamped the leg in place during the movement and accommodated a load cell (Kistler Instruments AG, Winterthur, Switzerland) for the measurement of the applied forces. In addition, an accelerometer (K-SHEAR; Kistler Instruments AG) was attached to the lower leg in order to measure the angular accelerations produced by the release. The Cybex setup and leg cuff are shown in Figures 1 and 2.

To illustrate the capability of the modified ergometer to measure SEC stiffness of the knee flexors, data collected on a single participant (height $175 \mathrm{~cm}$, mass 89.3 $\mathrm{kg}$, age $24 \mathrm{yrs}$ ) will be presented. Prior to participating in the study, the participant read and signed a written consent document and the study was approved by the university's institutional review board.

The data collection procedure employed was based on

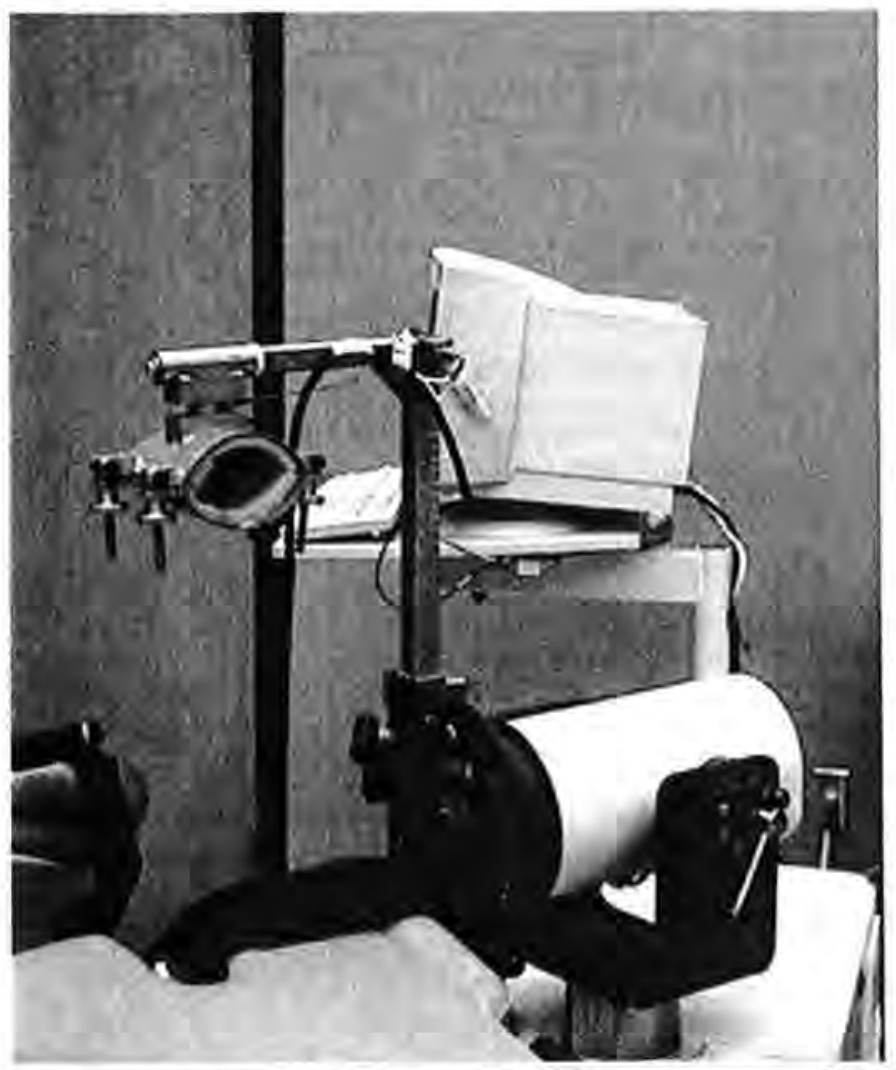

Figure 2. Ergometer used for controlled-release experiments.

the protocol described by de Zee and Voigt (3). In short, 3 types of release were performed: slow passive, fast passive, and fast active. In the 2 passive trials, the leg was moved through a $35^{\circ}$ range, from a starting angle of $15^{\circ}$ from full extension, of knee flexion while the leg muscles remained passive or inactivated. The slow passive trial consisted of moving the lower leg at approximately 0.1 $\mathrm{rad} \cdot \mathrm{s}^{-1}$, and the fast passive trial was performed at approximately $7.5 \mathrm{rad} \cdot \mathrm{s}^{-1}$. During the fast active trials, the participant produced various levels of isometric force against the leg cuff, which was subsequently released at approximately $7.5 \mathrm{rad} \cdot \mathrm{s}^{-1}$. The slow passive trial was used to correct for the passive stiffness of the knee flexors, and the fast passive trial was used to correct for inertial forces produced by the acceleration of the cuff and lower leg.

\section{Results}

The investigators determined that the customized ergometer was appropriate, according to the mechanical requirements outlined above, for measuring MTU stiffness of the knee flexors. An example of a moment-angle curve filtered and corrected for passive stiffness and inertial moments is shown in Figure 3. The final moment-angle curve produced by the shifting, averaging, and curve-fitting procedure is shown in Figure 4. The stiffness of the knee flexors was then calculated as the slope of the moment-angle curve and is plotted against moment in Figure 5. For the subject tested, the MTU stiffness of the knee flexors was $519 \mathrm{~N} \cdot \mathrm{m} \cdot \mathrm{rad}^{-1}$ at a knee flexion moment of $100 \mathrm{~N} \cdot \mathrm{m}$. 


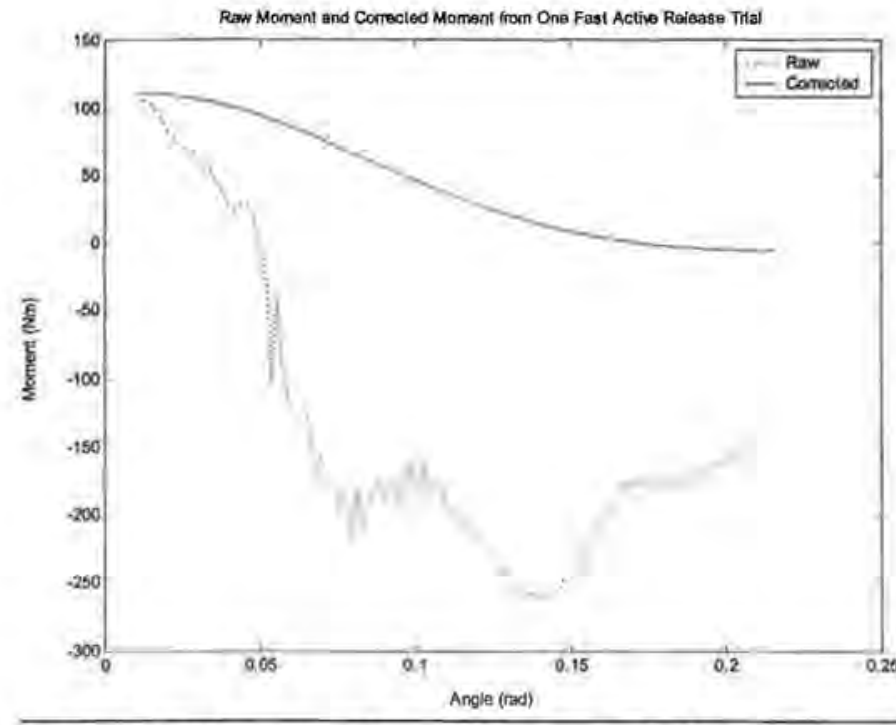

Figure 3. Moment-angle curve from a single fast-active release before and after correction for passive stiffness and inertial moment.

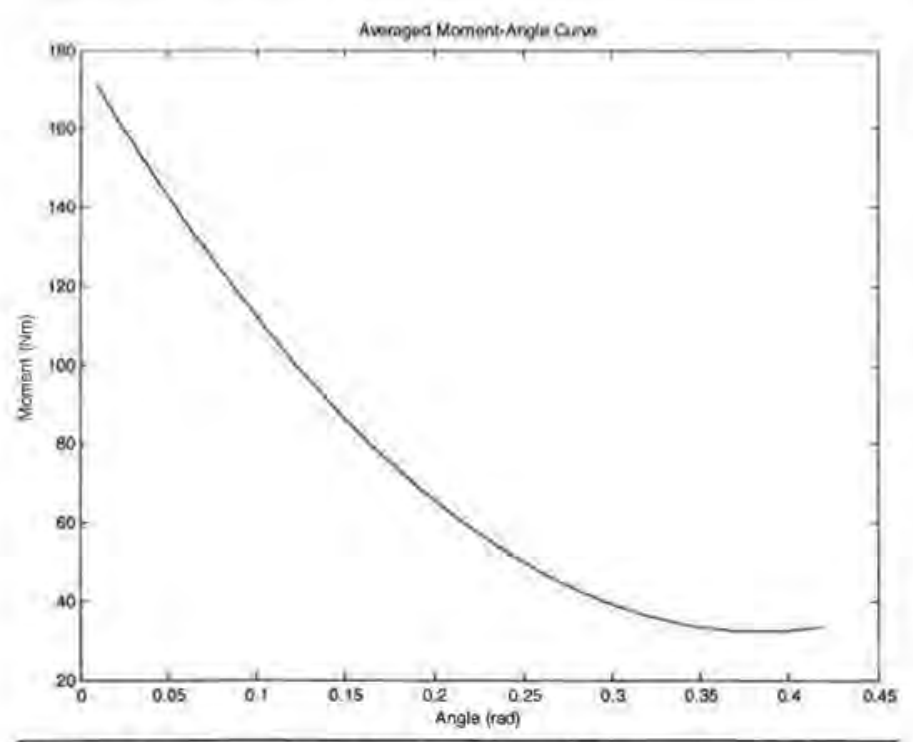

Frgure 4. The averaged moment-angle curve, for a representative participant, after all correction procedures, shifting, and curve-fitting procedures have been applied.

\section{Discussion}

The purpose of the study was to develop a device and methodology to measure the MTU stiffness of the knee flexors. This was achieved, and values within physiological range were obtained. Following is a discussion of the technical issues encountered in the course of developing this device and technique.

The data analysis procedures were adopted from those published by de Zee and Voigt (3) and Hof $(5,6)$. However, we encountered 2 problems that made the application of these procedures more difficult. The first was the obvious difference in limb size, foot versus lower leg and foot, and the subsequently higher inertial moments that had to be corrected. According to anthropometric data provided by Winter (15), the moment of inertia of the combined lower leg and foot is approximately 25 times greater than that

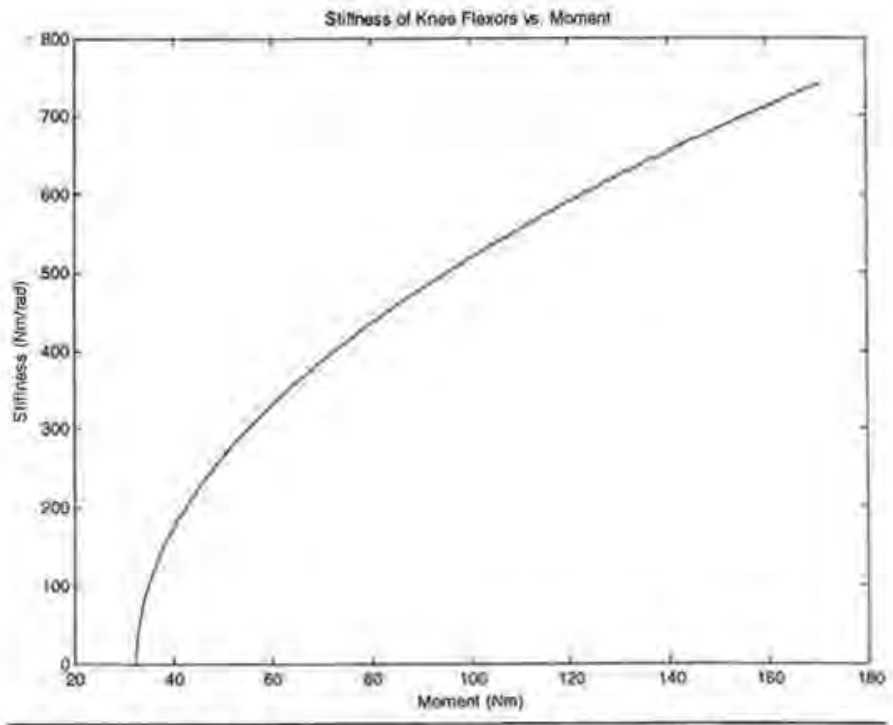

Figure 5. Series elastic component stiffness of the knee flexors vs. moment.

of the foot alone for an average-sized man. The second issue was the simple difference in the ergometers used. Hof and de Zee and Voigt both used a hydraulic ergometer, whereas we used a typical isokinetic ergometer. The Cybex NORM uses a BRU-200 brushless drive module to power and control an S-series permanent magnet synchronous motor equipped with an encoder for position measurement. This drive module and motor introduced a large amount of electrical noise that affected the signal clarity of the accelerometer and load cell used in the experiment.

The steps for correcting for the passive stiffness and inertial moments, as outlined by de Zee and Voigt, were applied to data obtained from the various releases with only minor modifications (3). The primary difference was the filtering cutoff used for the raw moment data from the fast passive and active trials. This data was low-pass filtered at $40 \mathrm{~Hz}$ using a fourth order Butterworth filter. It was determined, using a power spectrum density analysis of the signals, that this filter cutoff preserved the integrity of the signal while eliminating much of the electrical noise generated by the electronics of the Cybex.

As with the corrections for passive stiffness and inertial moments, we again followed the procedures outlined by de Zee and Voigt to angle-shift and average the corrected submaximal moment-angle curves. These shifts were necessary because each of the fast active releases began at a slightly different initial torque and, as demonstrated by de Zee and Voigt, it is justifiable to shift the submaximal curves to the right so that the initial moment corresponds to that of the release trial with the highest initial moment (3). Once the submaximal moment-angle curves were shifted, the average of the shifted curves was calculated and then a quadratic curve was fit to this averaged curve. The use of a quadratic fit of the data was based on the work of Hof, who reported that a quadratic fit of his data was as good as a quadratic plus linear model (6). The final moment-angle curve was produced by the shifting, averaging, and curve-fitting procedures (Figure 4). The stiffness of the knee flexors was then calculated as the slope of the moment-angle curve (Figure 5).

In addition to the correction and curve-fitting proce- 

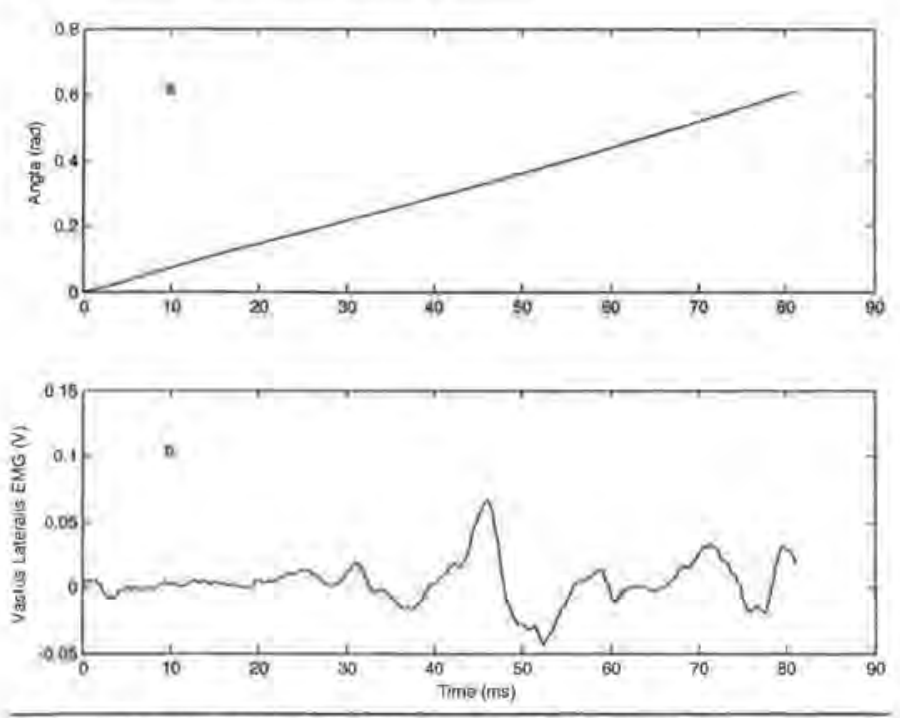

FigurE 6. (a) Angular displacement of ergometer arm measured by the built-in encoder. (b) Surface electromyography of the vastus lateralis band-pass filtered $(20-300 \mathrm{~Hz})$ from a representative fast active release.

dures outlined above, muscle activation of the biceps femoris (BF) and vastus lateralis (VL) were monitored using surface electromyography. Bursts of activity in either muscle group could have confounded the results obtained from this experiment. Although no increases in BF activity were found across the release trials, increases in VL activity, presumably caused by the stretch reflex, were evident in many trials between 40 and $50 \mathrm{~ms}$, as illustrated in Figure 6B.

Therefore, only data prior to initiation of the stretch reflex were used in the analysis, which is why the final moment-angle curve presented in Figure 4 does not include the entire $35^{\circ}$ of knee flexion used in the experiment.

The results presented here indicate that it is possible to perform a controlled-release measurement of the SEC stiffness of the knee flexors by modifying the capabilities of a commercial ergometer. The stiffness values presented for the knee flexors are higher than those reported by Hof and by de Zee and Voigt for the plantar flexors, $306 \pm 39$ and $506 \pm 72 \mathrm{~N} \cdot \mathrm{m} \cdot \mathrm{rad}^{-1}$ respectively, at a moment of 100 $\mathrm{N} \cdot \mathrm{m}$; this is to be expected, however, based on the function of the musculotendinous structures in question (3, $6)$. The functional and structural differences in leg muscle-tendon architecture is very well described by Alexander and Ker (1). The primary knee flexors, the hamstring muscles, have much longer fascicles and shorter tendons when compared to the plantar flexors of the ankle. These structural differences are closely tied to the functional differences of the 2 muscle-tendon groups, with the hamstring muscles generating large forces to accelerate or decelerate the body during activities such as running or jumping, whereas the plantar flexors and their longer tendons are more suited to store and release elastic energy during the same types of activities (1). In other words, it is expected that the plantar flexors would be more compliant than the knee flexors, based on the structural and functional differences of the 2 muscle groups. This was a potential obstacle for the use of the controlledrelease protocol, which is based on the measurement of the stored elastic energy of the tissue in question. However, despite this and the other technical difficulties, it is possible based on these results to measure the SEC elasticity of the knee flexors with such a protocol.

\section{Practical Applications}

This research has demonstrated that it is possible to refit a commercial ergometer, such as a Cybex, for the measurement of musculotendinous stiffness of the knee flexors during high loading conditions. However, this procedure does present some technical difficulties and will most likely require a combination of biomechanists, computer programmers, and engineering technology personnel to make the appropriate ergometer adaptations. Although these technical difficulties must be overcome, the knowledge that these commercial devices can be successfully refitted for this type of measurement may stimulate further research using this protocol. Using this technique, the role of the elastic characteristics of the knee flexors in performance and injury prevention may be examined in detail; this opens up an important avenue for scientific investigation with high relevance for the strength and conditioning field. Stiffness of the knee flexors may be an important factor in hamstring muscle strain, and it has been theorized that this mechanical factor is critical in controlling the anterior shift of the tibia during landings and therefore the risk of anterior cruciate ligament rupture. Although further research is required, the technique we have developed may prove an important screening tool to reduce the incidence of such injuries. In the near future, this technique should be applied to assess the efficacy of various strength and conditioning programs designed for prophylactic measures against ACL and hamstring injuries. It is important to note that the modification of this isokinetic device did not interfere with its standard use. It is hoped that this formative research will encourage equipment manufacturers to develop commercial versions for more widespread implementation in exercise, rehabilitation, and sports sciences.

\section{REFERENCES}

1. ALEXANDER, R.M., AND R.F. KER. The architecture of leg muscles. In: Multiple Muscle Systems: Biomechanics and Movement Organization. J.M. Winters and S.L.-Y. Woo, eds. New York: Springer-Verlag, 1990. pp. 568-577.

2. Butler, R.J., H.P. Crowell 3rd, and I.M. Davis. Lower extremity stiffness: Implications for performance and injury. Clin. Biomech. (Bristol, Avon) 18:511-517. 2003.

3. DE ZEE, M. AND M. VoIGT. Moment dependency of the series elastic stiffness in the human plantar flexors measured in vivo. J. Biomech. 34:1399-1406. 2001.

4. HoF, A.L. A controlled-release ergometer for the human ankle. J. Biomech. 30:203-206. 1997.

5. HoF, A.L. Correcting for limb inertia and compliance in fast ergometers. J. Biomech. 30:295-297. 1997.

6. HoF, A.L. In vivo measurement of the series elasticity release curve of human triceps surae muscle. J. Biomech. 31:793-800. 1998.

7. HoF, A.L. Muscle mechanics and neuromuscular control. $J$. Biomech. 36:1031-1038, 2003.

8. KuBo, K, Y. KaWAKami, AND T. FukUnaGa. Influence of elastic properties of tendon structures on jump performance in humans. J. Appl. Physiol. 87:2090-2096. 1999.

9. LATASH, M.L., AND V.M. ZATSIORSKY. Joint stiffness: Myth or reality? Hum. Mov. Sci. 12:653-692. 1993. 
10. MaHugh, M.P., D.A. ConNolly, R. G. Eston, I.J. KREMENic, B.J. NiChOLAS, AND G.W. GLEIM. The role of passive muscle stiffness in symptoms of exercise-induced muscle damage. Am J. Sports Med. 27:594-599. 1999 ,

11. McNaIR, P.J. AND R.N. MARSHALL. Landing characteristice in subjects with normal and anterior cruciate ligament deficient knee joints. Arch. Phys. Med. Rehabil. 75:584-589, 1994.

12. Pandy, M.G., AND K.B. Shrlburne. Dependence of cruciateligament loading on muscle forces and external load. $J$. Biomech. 30:1015-1024. 1997.

13. WALSHE, A.D., AND G.J. WIIsON. The influence of musculotendinous stiffiness on drop jump performance. Can. J. Appl. Phy. siol. 22:117-132. 1997.

14. Wirson. G.J., A.J. MurPhy, AND J.F. Pryor. Musculotendinous stiffness: Its relationship to eccentric, isometric, and concentric performance. J. Appl. Physiol. 76:2714-2719. 1994.
15. Winter, D.A. Biomechanics and Motor Control of Human Movement. 2nd ed. New York: John Wiley \& Sons Inc., 1990.

\section{Acknowledgments}

The authors would like to thank Gary Trobridge for his assistance in the design and manufacture of the custom leg cuff, We would also like to thank Zach Riley, Phil Newell, Melissa Colthrust, Sarah Kruger, and Jeff Frame for their time and assistance. This project was supported in part by the National Strength and Conditioning Graduate Student Grant.

Address correspondence to Dr. Eric L. Dugan, eldugan@ bsu.edu. 